\title{
Pobreza alimentaria: inseguridad y vulnerabilidad en las regiones de Sonora en 2015
}

\section{Food Poverty: Insecurity and Vulnerability in the Regions of Sonora in 2015}

\author{
Abel E. Castro Robles* \\ Centro de Investigación en Alimentación y Desarrollo, Hermosillo, Sonora, México \\ ORCID: http://orcid.org/0000-0003-3187-3499 \\ Mario Camberos Castro ${ }^{* *}$ \\ Centro de Investigación en Alimentación y Desarrollo, Hermosillo, Sonora, México \\ ORCID: http://orcid.org/o000-0002-327I-2980 \\ DOI: http://dx,doi.org/I0.28928/revistaiztapalapa/832017/atc2/castroroblesae/camberoscastrom
}

\begin{abstract}
Resumen:
En la actualidad el fenómeno de la inseguridad alimentaria se asocia con la pobreza extrema, que identifica a personas $y / o$ familias con ingresos insuficientes para adquirir los bienes necesarios que satisfagan los mínimos requerimientos nutrimentales; sin embargo, la inseguridad se refiere a aquellos individuos que aseguran haber experimentado la falta de calidad, cantidad o ingesta de nutrientes en alguna hora del día e incluso la falta de acceso a estos durante la jornada completa, hasta llegar a padecer hambre. Se trata de un problema esencialmente de carácter estructural, que supone la confluencia de factores económicos, demográficos, culturales, sociales y políticos, y que ha derivado en una situación compleja de indefensión, pobreza y creciente vulnerabilidad social. Si bien los pobres urbanos comparten carencias con los del medio rural, la ubicación geográfica es un componente clave para comprender la estructura, las causas y las tendencias de la pobreza en cada sector. En nuestro análisis planteamos como objetivo estimar cómo se presentan tales factores en las regiones de Sonora, así como las condiciones de vulnerabilidad que enfrenta esa población en lo tocante a temas de carencia educativa, inseguridad en el empleo, cobertura de los sistemas de seguridad y la pertenencia a algún grupo indígena, todo ello con base en la EMSA (Escala Mexicana de Seguridad Alimentaria) incluida en la Encuesta Intercensal 2015 (EIC-2015), donde la población en pobreza alimentaria por ingreso alcanzó un índice de $20.7 \%$ de la población sonorense, mientras que $27.88 \%$ experimentó falta de ingesta alimentaria.
\end{abstract}

Palabras clave: ingreso, acceso, estructural, EMSA, EIC-2015.

\begin{abstract}
At present the phenomenon of food insecurity is associated with extreme poverty, which identifies persons and / or families with insufficient income to acquire the necessary goods that meet the minimum nutritional requirements; However, insecurity refers to those individuals who claim to have experienced lack of quality, quantity or intake of food at any time of day and even lack of access to food throughout the day, until they become hungry. The problem of food and nutrition is essentially structural and depends on economic, demographic, cultural, social and political factors, which has fostered a complex situation of defenselessness, poverty and increasing social vulnerability. While the urban poor share gaps with their rural counterpart, geographical location is a key component in understanding the structure, causes, and trends of poverty. In our analysis we propose to estimate food poverty and insecurity or lack of access in the regions of Sonora; As well as the conditions of vulnerability faced by said population, related to lack of education, insecurity in employment, non-belonging to a security system and being part of some indigenous group, through the Mexican Food Security Scale (EMSA, for its acronym in Spanish) included In the Intercensal Survey 2015 (EIC-20I5 for its acronym in Spanish). Where the population in food poverty by income registered $20.7 \%$ while access insecurity reached $27.88 \%$ of the population of Sonora who experienced the lack of food intake. Key words: Income, Access, Structural, EMSA, EIC-20I5
\end{abstract}


$E$ 1 fenómeno actual de inseguridad alimentaria se asocia con la pobreza extrema, o pobreza alimentaria, que identifica a personas o familias con ingresos insuficientes para alcanzar el mínimo de requerimientos nutrimentales cotidianos recomendados. La inseguridad se refiere a aquellos que declaran haberla experimentado en algún grado ya sea por la falta de calidad o por la cantidad exigua de alimento a su alcance en alguna hora del día, e incluso por un nulo acceso a satisfactores de ese tipo durante la jornada, hasta llegar a padecer hambre.

Ante el cometido de alcanzar la seguridad alimentaria mundial — que todas las personas, en todo momento, tengan acceso a los alimentos-, nueva meta del milenio de la Organización de las Naciones Unidas para la Alimentación y la Agricultura (FAO, 2002, 2015), se reconoce que existen dos restricciones: la física, que es la disponibilidad, y la económica, que es la percepción de un ingreso suficiente para adquirir en el mercado la canasta llámese básica o normativa con alimentos suficientes, inocuos y nutritivos para satisfacer las necesidades y preferencias de cada persona según los estándares de instituciones globales como la FAO (20IO), a nivel mundial, y del Instituto Nacional de Ciencias Médicas y Nutrición Salvador Zubirán (INNSZ, 2003), de México (Plan Nacional de Desarrollo 2013-2018).

Se ha documentado que la pobreza en el país rebasa el problema de la alimentación y la nutrición, y se define esencialmente por su carácter estructural y asociado a factores económicos, demográficos, culturales, sociales y políticos, todo lo cual es prueba de que el modelo económico actual y sus políticas sociales han promovido una situación compleja de indefensión, precariedad y creciente vulnerabilidad social (Castillo y Arzate, 2013), porque ya no se trata de una situación de contingencia sino del preocupante círculo vicioso de la pobreza, razón por la cual hay que investigar más a fondo.

Aunque se advierten condiciones de carencia semejantes entre los pobres urbanos y los rurales, la ubicación geográfica es un componente clave para comprender la estructura, las causas y las tendencias de la pobreza, la vulnerabilidad y el hambre. Y para luchar contra estos flagelos es necesario poner en práctica políticas enfocadas (CONEVAL, 2OI2) que permitan romper con la transmisión generacional. 
En el presente trabajo se adopta el "enfoque monetario de la pobreza" o método de líneas de pobreza (Sen, 1976) y el precepto de acceso a la alimentación -Escala Mexicana de Seguridad Alimentaria (emSA) -, para identificar, en Sonora, en 2015, aquellas viviendas particulares habitadas cuyos moradores se hallan en pobreza alimentaria.

Este estudio presenta un enfoque novedoso, respecto de otros dedicados al problema de la pobreza o la inseguridad alimentaria, en el tema de la vulnerabilidad, cuya revisión supone aspectos como la carencia de instrucción educativa básica, la falta de prestaciones sociales y pensión, así como de empleo remunerado y la pertenencia a algún grupo indígena, que por lo demás hace más difícil que la población supere las situaciones de precariedad señaladas. Finalmente llegamos a las conclusiones.

Este análisis también tiene como propósito responder a las siguientes preguntas:

- ¿Es la inseguridad alimentaria privativa de la pobreza por ingreso?

- ¿ Los mayores niveles de pobreza e inseguridad alimentaria corresponden a la región rural?

- ¿La región con mayor concentración poblacional encabeza también los niveles de pobreza y de inseguridad alimentaria?

- ¿La vulnerabilidad de la población en pobreza alimentaria se restringe a aquellos con menores niveles de educación y ausencia de seguridad social?

Para responder estas y otras incógnitas implícitas en nuestro análisis planteamos como objetivo estimar: pobreza alimentaria, inseguridad o falta de acceso y condiciones de vulnerabilidad en términos de carencia de educación básica, inseguridad en el empleo, no pertenencia a un sistema de seguridad y ser integrante de algún grupo indígena.

Inicialmente presentamos una discusión acerca de los conceptos de pobreza alimentaria y su utilización; luego nos referimos a los métodos de líneas de pobreza (LP) y la Escala Mexicana de Seguridad Alimentación (emsa), el Coeficiente de Cronbach para la validación de la escala y la encuesta intercensal (EIC, 20I5) del INEGI.

En seguida aplicamos los métodos mencionados para la identificación de viviendas y habitantes en pobreza alimentaria por ingresos (PZAXY); asimismo, aquellas en pobreza alimentaria por inseguridad o acceso (PZAXACC), donde se interpretan los resultados e identifican las características que tienen en común de acuerdo con su distribución regional.

Posteriormente se caracterizan las distintas formas de vulnerabilidad que sufren los pobres alimentarios o por inseguridad y que hace más difícil superar su situación. 
Es importante destacar que no existe información para años anteriores que permitan integrar y evaluar todas las regiones en función de la pobreza e inseguridad alimentaria con todas las variables aquí señaladas, razón por la cual realizamos un análisis de las regiones de Sonora tipo corte transversal y presentamos al final conclusiones.

\section{Pobreza alimentaria, inseguridad y vulnerabilidad}

Normalmente, lo que se conoce como pobreza extrema es el porcentaje de personas cuyo ingreso está por debajo de la línea de pobreza (LP), lo cual hace referencia al valor monetario suficiente para cubrir las necesidades básicas de nutrición. En este sentido, la construcción de la canasta alimentaria es la forma más común que se ha utilizado para estimar el valor de dicha línea, y de ahí que la importancia de fijar un nivel básico de consumo radique en su efecto directo al medir pobreza.

Uno de los rasgos particulares de las canastas alimentarias es que pueden basarse en los mínimos de consumo para mantener una vida sana, a lo que se llama canasta normativa, y también considerando el dato real de la gente al tomar en cuenta el patrón de las familias registrado en la Encuesta Nacional de Ingreso-Gasto de los Hogares (ENIGH, 2015). Cabe mencionar lo anterior ya que la metodología utilizada por el coneval (20IO) es la de una canasta seminormativa, es decir, que toma en cuenta los patrones de consumo para determinar los bienes contenidos en la canasta alimentaria, pero ajusta las cantidades de tal forma que permitan un consumo adecuado de nutrientes.

La estimación de canastas básicas alimentarias a partir de la estructura de consumo de una sociedad es una alternativa ampliamente utilizada en México y otros países para realizar mediciones de pobreza. ${ }^{1}$ La ventaja es que permiten identificar la cantidad de dinero necesaria para satisfacer una de las más elementales necesidades humanas, la alimentación (Minor y Balcázar, 2014).

El estudio se fundamenta en el "enfoque monetario de la pobreza" o paradigma de la pobreza de ingresos, el cual supone que las diferencias en el bienestar de la población se pueden resumir por las diferencias entre ingreso y gastos; es decir, los pobres se definen por la condición de insuficiencia de ingresos para cubrir el costo de los satisfactores básicos. Ello implica que todas las personas transforman los

1 La pobreza extrema es equivalente a la pobreza alimentaria. 
ingresos en bienestar de igual manera; todos los hogares o personas se suponen idénticos (Camberos, Bracamontes y Gutiérrez, 20I2).

Además, el derecho a una alimentación adecuada es considerado como una condición indispensable en la seguridad alimentaria. La Organización de las Naciones Unidas para la Agricultura y la Alimentación (FAO, 2OII) entiende por seguridad alimentaria "cuando todas las personas tienen en todo momento el acceso físico, social y económico a alimentos suficientes, inocuos y nutritivos que satisfacen sus necesidades y preferencias alimentarias para llevar una vida activa y sana". Situaciones de inseguridad alimentaria se pueden producir debido a problemas de acceso físico o económico a los satisfactores o a los recursos o ingresos necesarios para adquirirlos, a la falta de disponibilidad y/o a un uso inadecuado de los alimentos.

En materia de disponibilidad, México no enfrenta problemas de insuficiencia global (Camberos, 2000). Según indicadores de la FAO, el suministro interno para la población general es muy superior al necesario. Desde hace más de 40 años se alcanzó el nivel de suministro de energía per cápita mínimo indispensable (2 600 kilocalorías) para satisfacer los requerimientos de toda la población; según reportan las hojas de balance de la FAO, para 2005 se había llegado en el país a 3270 kilocalorías (CONEVAl, 20I2), el nivel más alto en América Latina.

Antes que un conflicto de disponibilidad, por tanto, los problemas de inseguridad alimentaria en México se relacionan con el acceso y la capacidad económica de la población para adquirir, comprar o producir suficientes y variados alimentos que les permitan cubrir sus necesidades básicas de nutrición (IPEA, 2002, en ABRANDH, 20I0; Camberos, 2000; Maluf y otros, 1996; Hoffman, 1994). La FAo ha señalado reiteradamente que la seguridad alimentaria es principalmente un problema de acceso, que afecta en mayor medida a las personas en condición de pobreza y, particularmente, de extrema pobreza. El asunto tiene que ver básicamente con el hecho de que los ingresos de una parte de la población resultan insuficientes para adquirir alimentos y asegurar de este modo una dieta adecuada (Gómez, 2013: 55-68).

\section{Metodología para medir pobreza alimentaria e inseguridad}

La forma de medir la pobreza puede de una manera importante influir en el cómo llegamos a entenderla, cómo la analizamos y cómo creamos políticas para incidir en ella. Por esta razón las metodologías de mensura pueden ser de gran relevancia práctica (Alkire y Foster, 20II). Desde Sen (1976), la medición de la pobreza ha sido conceptualizada en dos principales pasos: identificación de quién es pobre $y$, 
agregación de la información acerca de la pobreza entre la sociedad. En el espacio unidimensional por ingreso, la identificación de quién es pobre es relativamente clara. Con una línea de pobreza - determinada por el ingreso necesario para adquirir una canasta básica de bienes y servicios- para diferenciar la población entre los pobres y los no pobres (Camberos et al., 1996).

\section{El método de pobreza por ingresos para medir la pobreza alimentaria}

El método de líneas de pobreza (LP) desarrollado de manera formal por Amartya Sen (1976), es un método indirecto, pues se identifica a los hogares y a las personas pobres a partir de si pueden o no satisfacer un conjunto de necesidades básicas en función de su ingreso; es decir, se compara el ingreso corriente de los hogares y las personas contra una línea de pobreza especificada (Damián et al., 2004). De esta manera, el método de LP toma como referencia el costo de los satisfactores básicos o mínimos llamado línea de pobreza $(Z)$, por lo que se consideran en pobreza a aquellas familias o individuos cuyo ingreso $\left(\mathrm{Y}_{i}\right)$ está por debajo de la línea de pobreza $(\mathrm{Z})$. Entonces, si se cumple la condición: $\left(Z-Y_{i}\right)>0$, el hogar será pobre $\left(n_{i}\right)$ y el total de hogares en pobreza será $\left(\mathrm{N}_{i}\right)$ (Camberos et al., 2013).

Foster, Greer y Thorbecke (1984) generalizan el método LP introduciendo un ponderador " $\alpha$ ", el cual es un factor de valoración subjetiva del grado de pobreza:

Ecuación. I

$$
\operatorname{FGT}(\alpha)=\mathrm{P}_{\alpha}(y, z)=\frac{1}{\mathrm{~N}} \sum \begin{aligned}
& \mathrm{i}=\mathrm{q} \\
& \mathrm{i}=1
\end{aligned}\left[\frac{z-y_{\mathrm{i}}}{z}\right]^{\alpha}
$$

Si el parámetro $\alpha=0$, el índice FGT (o) mide el porcentaje o la incidencia de hogares en pobreza; mientras que, si $\alpha=$ I el índice FGT (I) representa la brecha agregada y FGT (2) la severidad de la pobreza que sirve para identificar a los más pobres de los pobres.

\section{La escala de medición de la inseguridad alimentaria o acceso}

Para una correcta medición y estimación de la pobreza alimentaria y ante el cometido de que todas las personas, en todo momento, tengan acceso físico y económico a suficientes alimentos inocuos y nutritivos para satisfacer sus necesidades y preferencias de alimentación, con el fin de llevar una vida activa y sana y gozar de una 
seguridad alimentaria (FAO, 2OII, CONEVAL, 20I4), se manejará una escala que nos permita observar el acceso y la experiencia que se presentan en las viviendas y sus habitantes frente a este fenómeno.

En México se ha utilizado, entre las encuestas de hogares, la Escala Latinoamericana y Caribeña de Seguridad Alimentaria (elCSA), elaborada por la FAo, la cual ofrece mediciones basadas en la experiencia. Esta metodología, asumida por el coneval para estudiar la situación del país, ha dado como resultado la Escala Mexicana de Seguridad Alimentaria (EMSA). A raíz de lo anterior, con base en la EMSA y siguiendo las recomendaciones del CONEVAL (20I4), la metodología se complementa con la Encuesta Intercensal 2015 (EIC-I5) (Castro, 2016), con las cuales se elaboran escalas de percepción y se prueba su consistencia interna para poder ser aplicada con propiedad.

La consistencia interna de una escala se refiere a qué tan reproducibles son los patrones de respuesta entre individuos. Si la consistencia interna es adecuada, entonces ese patrón de respuesta debe poder reproducirse cuando se seleccionan más individuos para responder a las preguntas de la misma escala (ELCSA, 2OI2).

En este trabajo se utilizó el coeficiente alfa de Cronbach, ${ }^{2}$ que se define como la correlación promedio entre los ítems del instrumento. Puede adoptar valores entre o y I. Estimaciones entre 0.70 y 0.90 indican que la consistencia interna es adecuada, es decir, la escala evalúa correctamente el constructo teórico que busca medir. Valores superiores a 0.90 sugieren redundancia o duplicación.

Se estimó el coeficiente para dos encuestas 2015 y 20I0. En el cuadro I se muestran los resultados con valores de Cronbach 0.92, solamente válidos cuando no se incluye a menores de edad, o sea, solo se considera a mayores de edad. La aplicación a la encuesta 20 Io arrojó resultados muy cerca de I, por lo que no se incluye en este trabajo (para un desarrollo completo de la metodología véase Castro, 20ı6).

2 Técnica utilizada en la elCSA 2012 para analizar la consistencia interna de la seguridad alimentaria; en este trabajo se complementó también con la prueba del ordenamiento de las preguntas y el análisis de prevalencia de respuestas (Villagómez-Ornelas, Hernández-López, Carrasco-Enríquez, Barrios-Sánchez, Pérez-Escamilla y Melgar-Quinónez, 2014). Además, se aplicó el Criterio Bayesiano de Información BIC y el Criterio Akaikeano de Información AIC. En su conjunto estas técnicas arrojaron resultados que muestran la fortaleza del constructo, donde el conjunto de las preguntas de la EMSA cumple adecuadamente al identificar aquellas viviendas cuyos ocupantes presentan el atributo de interés (la seguridad o inseguridad alimentaria en este caso) y medir con ella la intensidad con la que experimentan el fenómeno. 


\section{Cuadro i. Coeficiente alfa de Cronbach para hogares CON O SIN MENORES EN LA EIC-2OI5.}

\begin{tabular}{lccc}
\hline & Con menores & Sin menores & Solo menores \\
\hline Promedio de covarianzas intre ítems & 0.72 & 0.75 & 0.06 \\
Número de ítems en la escala & $\mathrm{I} 2$ & 6 & 6 \\
Coeficiente de confiabilidad de la escala & 0.97 & 0.92 & 0.92 \\
\hline
\end{tabular}

Fuente: Estimaciones propias con base en EIC-I5, EMSA y Coef. de Cronbach, con el programa Stata 2013.

\section{Aspectos socioeconómicos y regionalización del Estado}

Para dimensionar la entidad en el ámbito nacional ofrecemos estadísticas comparativas sobre su población, participación económica medida por el PIB, nivel educativo, analfabetismo, en las dos décadas transcurridas del siglo XXI.

\section{Características socioeconómicas}

Según los resultados de los Censos de Población y Vivienda, en 1990 Sonora alcanza una población de I 823 606; en 2000, de 2216969 y en 2010 reporta 2662480 habitantes, lo cual representa una tasa media de crecimiento de I.9\% para este periodo de 20 años, por encima de la tasa media de crecimiento poblacional nacional (I, $8 \%$ ). Para 2015 se calculaban 2850330 habitantes en dicha entidad, con una tasa de crecimiento del I.37\% similar a la nacional (I.4\%).

El Estado contribuyó al (PIB) durante los años de referencia (20IO y 2015) con $2.57 \%$ y $2.94 \%$, respectivamente, en tanto que su contribución a la PEA ocupada fue de $2.45 \%$ y $2.90 \%$, lo cual indica que fue ligeramente más productiva que el promedio de la PEA nacional, y eso se ve reflejado en el PIB per cápita, el cual también es superior para Sonora que el promedio nacional (INEgI, 20I5). También es de destacar que los porcentaje de personas afiliadas a servicios de salud y de hogares con jefatura femenina son mayores en el Estado que a nivel nacional.

Por otro lado, en términos de educación y etnicidad se aprecia un mejor escenario y desarrollo a nivel local que en lo nacional, ya que el porcentaje de asistencia de menores a la escuela es mayor en Sonora que en México y, como resultado, la población adulta registra hasta un segundo grado de educación media superior y el 
promedio de analfabetismo local equivale a poco menos de la mitad $(2.2 \%)$ del rango nacional (5.5\%). Esto da muestras de un mejor desarrollo y crecimiento económico en la entidad en comparación con la perspectiva nacional del periodo 2010-2015, en cuanto a vivienda, empleo, salud, educación y bienestar (PIB per cápita) se refiere, ${ }^{3}$ lo cual haría suponer niveles bajos de pobreza, inseguridad y vulnerabilidad.

\title{
Regionalización
}

A fin de identificar las características comunes de la población inmersa en la pobreza e inseguridad alimentaria, se adoptó la regionalización del Comité de Planeación para el Desarrollo del Estado de Sonora (Coplades), la cual hace referencia a la agrupación municipal según características sociodemográficas, económicas, hidrológicas y de tipo y uso de suelo en el Estado (Camberos 1994; 1996). Asimismo, como lo mencionamos con antelación, consideramos el análisis por zona (urbana y rural), ya que estas poseen rasgos singulares en Sonora.

La entidad se divide en I2 regiones (Camberos et al., 1996), a saber:

\section{Figura i. Regionalización de Sonora, 2015}

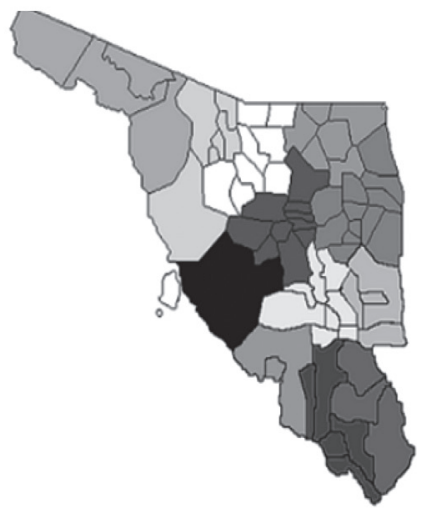

\author{
Región Desierto \\ Región Rio Altar \\ Región Frontera Centro \\ Región Frontera Norte \\ Región Rio Sonora y San Miguel \\ Región Sierra Alta \\ Región Hermosillo \\ Región Centro \\ Región Sierra \\ Región Guaymas-Empalme \\ Región Yaqui-Mayo \\ Región Sierra Baja
}

3 Elaboración propia con base en Inegi; Iter del Censo de Población y Vivienda 20Io, Encuesta Intercensal 2015, PIв y Cuentas Nacionales e indicadores de ocupación y empleo 2015. 


\section{Regiones de Sonora:}

Región Centro¥ 021 La Colorada, 037 Mazatán, 044 Ónavas, 054 San Javier, 057 San Pedro de la Cueva, 06r Soyopa, 062 Suaqui Grande y 068 Villa Pesqueira;

Región Desiertoः 017 Caborca, 055 San Luis Río Colorado, 048 Puerto Peñasco y 070 General Plutarco Elías Calles;

Región Frontera Centro: o16 Benjamín Hill, 022 Cucurpe, 035 Ímuris, 036 Magdalena, 043 Nogales, 058 Santa Ana, 059 Santa Cruz y 064 Trincheras;

Región Frontera Norteः 002 Agua Prieta, oII Bacoachi, org Cananea, 027 Fronteras, 039 Naco 04I y Nacozari de García;

Región Guaymas-Empalmeः 025 Empalme, 029 Guaymas y 072 San Ignacio Río Muerto;

Región Hermosillo: 030 Hermosillo;

Región Río Altar: 004 Altar, 007 Átil, 046 Oquitoa, 047 Pitiquito, 060 Sáric y 065 Tubutama;

Región Río Sonora y San Miguelः o01 Aconchi, o06 Arizpe, o13 Banámichi, o14 Baviácora, 020 Carbó, 034 Huépac, 045 Opodepe, 050 Rayón, 053 San Felipe de Jesús, 056 San Miguel de Horcasitas y 066 Ures;

Región Sierra: 005 Arivechi, 009 Bacanora, 052 Sahuaripa y 069 Yécora;

Región Sierra Alta: 008 Bacadéhuachi, oro Bacerac, 015 Bavispe, 023 Cumpas, 024 Divisaderos, 028 Granados, 03I Huachinera, 032 Huásabas, 038 Moctezuma, 040 Nácori Chico, 063 Tepache y 067 Villa Hidalgo;

Región Sierra Baja: 003 Álamos, 049 Quiriego y 05I Rosario y;

Región Yaqui-Mayo: o12 Bácum, or8 Cajeme, o26 Etchojoa, 033 Huatabampo, 042 Navojoa y 07I Benito Juárez.

Fuente: Camberos (1994, 1996) con base en Coplades, Plan Estatal de Desarrollo Sonora (1980; 1986).

\section{Pobreza alimentaria e inseguridad regional}

Una vez aplicadas las técnicas de identificación de la pobreza por ingreso y acceso, en el cuadro 2 se muestran los resultados para cada una de las regiones, que incluye la población total y la población en pobreza alimentaria por ingreso y por acceso. 


\section{Estimación para las regiones}

La región de Hermosillo concentra 32.26 \% de la población estatal, seguida por la Yaqui-Mayo con 27.38\%, luego la región Desierto con $11.76 \%$; después, Frontera Centro con 10.96\%; Guaymas-Empalme, con 7.93\%, ocupa la quinta posición; en sexto lugar está Frontera Norte, mientras que Río Altar contribuye con 2.07\%, Sierra, Sierra Alta y Sierra Baja en conjunto, con 2.II \%; la región Centro apenas alcanza $0.032 \%$.

Cuadro 2. Distribución regional de la población, Según CONDICIÓn De pobreza e INSEguridad alimentaria en Sonora 2015.

\begin{tabular}{|c|c|c|c|c|c|c|}
\hline \multirow[t]{3}{*}{ REGIONES } & \multicolumn{3}{|c|}{ Población } & \multicolumn{3}{|c|}{ Porcentaje } \\
\hline & \multirow{2}{*}{ Total } & \multicolumn{2}{|c|}{ Pobreza por } & \multirow{2}{*}{ Total } & \multicolumn{2}{|c|}{ Pobreza por } \\
\hline & & Acceso & Ingreso & & Acceso & Ingreso \\
\hline Centro & 7822 & I 477 & 969 & 0.32 & $0.2 \mathrm{I}$ & 0.19 \\
\hline Desierto & 290601 & 9I I33 & 65207 & II.76 & 13.23 & $\mathrm{I} 2.74$ \\
\hline Frontera Centro & 270919 & 65877 & 53583 & 10.96 & 9.56 & 10.47 \\
\hline Frontera Norte & 129190 & 28327 & 27708 & 5.23 & $4 \cdot \mathrm{II}$ & 5.42 \\
\hline Guaymas-Empalme & 195906 & 65021 & 48472 & 7.93 & 9.44 & 9.47 \\
\hline Hermosillo & 797429 & I9I 806 & IO7 I84 & 32.26 & 27.83 & 20.95 \\
\hline Río Altar & 19167 & 4157 & 3222 & 0.78 & 0.60 & 0.63 \\
\hline Río San Miguel & 31 967 & 8951 & 5000 & 1.29 & I.3O & 0.98 \\
\hline Sierra & 9473 & 2279 & 2107 & 0.38 & 0.33 & $0.4 \mathrm{I}$ \\
\hline Sierra Alta & 17238 & 3868 & 3467 & 0.70 & 0.56 & 0.68 \\
\hline Sierra Baja & 25388 & 8358 & 8418 & $\mathrm{I} . \mathrm{O} 3$ & $\mathrm{I} .2 \mathrm{I}$ & I. 65 \\
\hline Yaqui-Mayo & $6767 \mathrm{II}$ & 217841 & 186292 & 27.38 & $31.6 \mathrm{I}$ & $36.4 \mathrm{I}$ \\
\hline Total & 247 I 8II & 689095 & 511629 & 100 & 27.88 & 20.70 \\
\hline
\end{tabular}

Fuente: Cálculos propios con información de la encuesta intercensal 2015 INEGI. 
La columna 6 se refiere al porcentaje de la población en pobreza alimentaria por acceso y señala que la región Yaqui-Mayo concentra 31.6I \% de sus habitantes inmersos en esta problemática, seguida por Hermosillo con $27.83 \%$; después, Desierto con I3.23\%; mientras que Frontera Centro y Norte registran juntas $13.67 \%$; Guaymas-Empalme representan 9.44\%, cuando las regiones Río San Miguel y Río Altar registran I.9\% en esta condición; asimismo, Sierra, Sierra Alta y Baja figuran con I. 8 \% y la región Centro reporta $0.21 \%$ de sus habitantes en pobreza alimentaria por acceso.

Es de esperarse que a mayor concentración de población por región, esta refleje proporcionalmente el mismo peso relativo de la población con pobreza alimentaria por ingreso; sin embargo, en la columna 7 del cuadro 2 se observa en la región Yaqui-Mayo el porcentaje más alto, con 36.4I \%, muy por arriba de la región Hermosillo que concentra a $20.95 \%$ de sus habitantes en esta condición, cuando la de Hermosillo centraliza 32.26 \% de la población total en el Estado; Desierto, Frontera Centro, Guaymas-Empalme y Frontera Norte conservan esa representatividad y orden jerárquico poblacional, al igual que las regiones Centro, Sierra, Río Altar, Sierra Alta, Río San Miguel y Sierra Baja con menor representatividad y se enlistan de menor a mayor.

\section{Pobreza e inseguridad alimentaria de la población económicamente activa por zona urbana, rural y regiones}

Resulta muy importante considerar la condición de actividad laboral de la población en pobreza alimentaria por acceso e ingreso en el mercado de trabajo regional, dado que ahí se vierten los conocimientos, habilidades, experiencias y, como consecuencia, se refleja la diferencia estructural y regional del mercado laboral y, por lo tanto, esta parte contiene a la población en edad de trabajar que registró alguna actividad, buscó trabajo o simplemente no trabajó.

Para efectos de un mejor manejo, la información referida a la población en edad de trabajar se dividió en dos secciones, según la condición de pobreza alimentaria (ingreso y acceso) y por zona (rural y urbana). La primera parte se refiere a la población en pobreza alimentaria por ingreso y la segunda, a la población en pobreza alimentaria por acceso; en ambos casos la línea continua representa en la figura a la población que sí trabajó durante el periodo de levantamiento de la EIC-20I5, y las barras aluden a aquellas personas que no trabajaron y/o buscaron trabajo.

De un universo de I 920619 personas en edad de trabajar, $18.78 \%$ se encuentra en pobreza alimentaria por ingreso ( 360682 habitantes), pero $7.43 \%$ sí trabajaron, 
Figura 2. Población en edad de trabajar en pobreza alimentaria POR INGRESOS, SEGÚN ZONA Y CONDICIÓN DE ACTIVIDAD en las Regiones de Sonora, 2015.

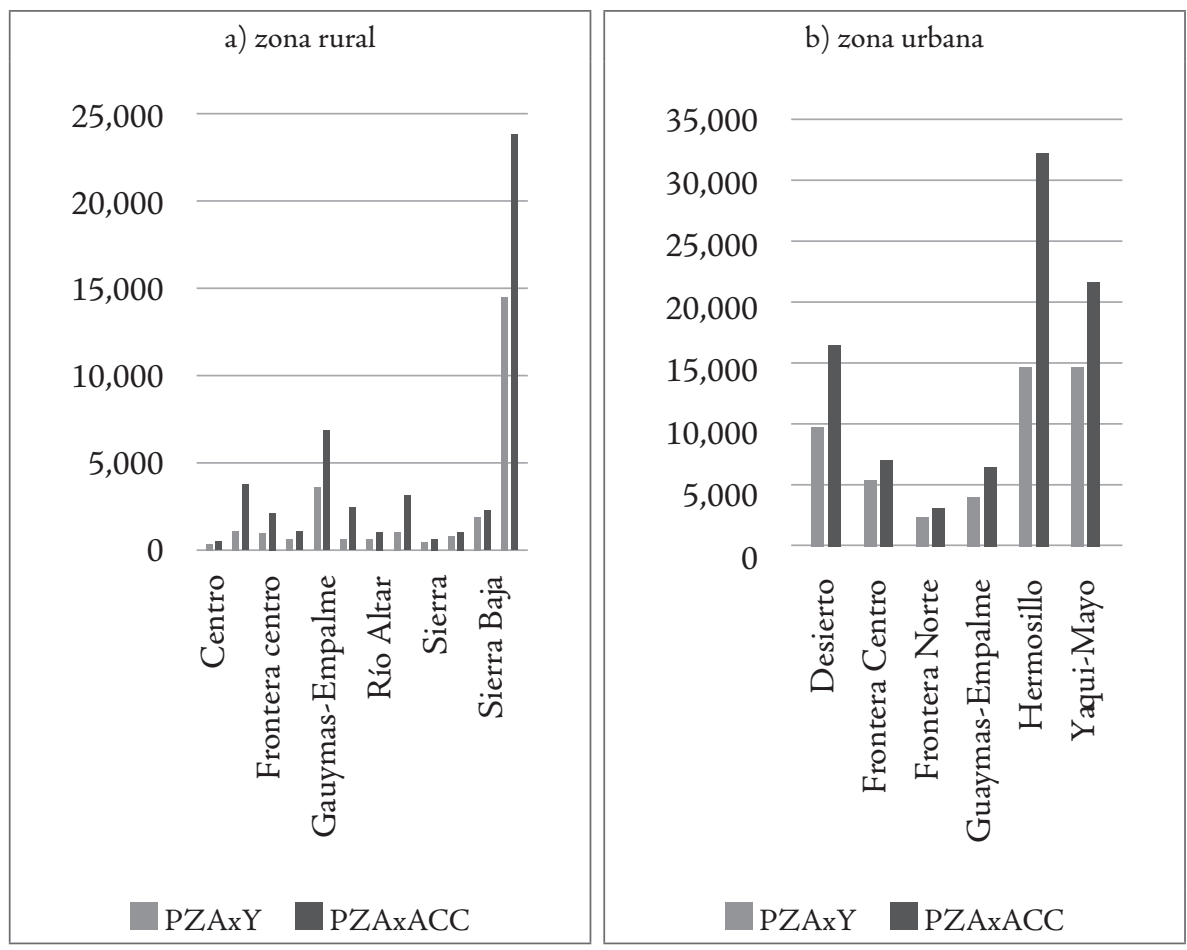

Fuente: Elaboración propia con base en la EIC-20I5, INEGI.

mientras que $3.79 \%$ no lo hicieron, cuando solo I.IO\% buscaron empleo. De la misma forma, 523575 habitantes se hallan en pobreza alimentaria por acceso (27.26\%), de los cuales $14.86 \%$ sí trabajaron, I. $44 \%$ no lo hicieron y $0.57 \%$ buscaron trabajo. Es importante destacar de este resultado que el porcentaje de la población en inseguridad que trabajó es el doble respecto de la que está en pobreza alimentaria, lo cual significa que su situación alimentaria es peor y por ello requeriría mayor atención.

$\mathrm{Al}$ hacer nuestro análisis por zonas, en la figura 2 se observa la zona rural en pobreza alimentaria por ingreso con cerca de 94879 personas, con $36.83 \%$ de personas que sí trabajaron (pero que no les alcanzó el ingreso para adquirir la canasta 
alimentaria), 9.08\% que no lo hicieron y I.46\% que buscaron empleo dentro de la zona rural sonorense, siendo la región Yaqui-Mayo la de la mayor cantidad de individuos que aunque trabajaron, se encuentran en pobreza alimentaria.

En lo que respecta a la zona urbana (en el cuadrante derecho de la figura 2) también es la región Yaqui-Mayo la que muestra la mayor cantidad de población que trabajó pero que está en pobreza alimentaria, seguida por Hermosillo (a pesar de que este municipio tiene mayor cantidad de población), y luego por las regiones Desierto, Frontera Norte, Guaymas-Empalme y Frontera Centro.

La dimensión de inseguridad alimentaria o pobreza por acceso, figura 3, muestra a 523575 habitantes en dicha situación, correspondiendo a la zona rural I 47566 personas, de las cuales $50.9 \%$ realizaron alguna actividad, $6.59 \%$ no trabajaron y I.I4 \% buscaron trabajo. La zona urbana compuesta por 376009 personas de las cuales $55.9 \%$ sí trabajaron, $4.79 \%$ no lo hicieron y $2.48 \%$ buscaron empleo; esto significa que en ambas zonas, la mitad de la población que está en inseguridad aun y que trabajó, representa un porcentaje mucho mayor que el que se encuentra en pobreza alimentaria por ingreso, de aproximadamente $36 \%$.

Para terminar este punto, en la figura 3 se observa que la región Yaqui-Mayo conjuntamente urbana-rural es la que concentra la mayor cantidad de población en inseguridad alimentaria, tal y como sucedió con la pobreza alimentaria por ingreso.

Cabe decir que este resultado ha sido una constante en los estudios del CIAD realizados por Camberos, Bracamontes y Huesca (1994, 1996, 2012 y 2013), sobre la pobreza en las regiones de Sonora en las últimas tres décadas, y es preocupante porque cuestiona la efectividad de los programas federales y locales de combate a la pobreza implementados en la región desde hace cinco sexenios, orientados a reducirla y a evitar su transmisión generacional.

\section{Vulnerabilidad de la población en condiciones de pobreza e inseguridad alimentaria}

Hemos visto el estatus que mantienen las regiones en relación con distintas variables como zona e (in)seguridad alimentaria de la población en pobreza alimentaria por acceso e ingreso. Ahora distinguiremos entre la población en estas condiciones aquella que consideramos se torna más vulnerables para el desarrollo de las regiones, toda vez que a su condición de pobreza se agregan carencias de capacidades, falta de prestaciones y condición étnica que les impedirá salir de la pobreza, porque se encuentran inmersos no en una situación, sino en el círculo vicioso de la pobreza. 
Figura 3. Población en edad de trabajar en pobreza alimentaria POR ACCESO, SEGÚN ZONA Y CONDICIÓN DE ACTIVIDAD

en las Regiones de Sonora, 2015.

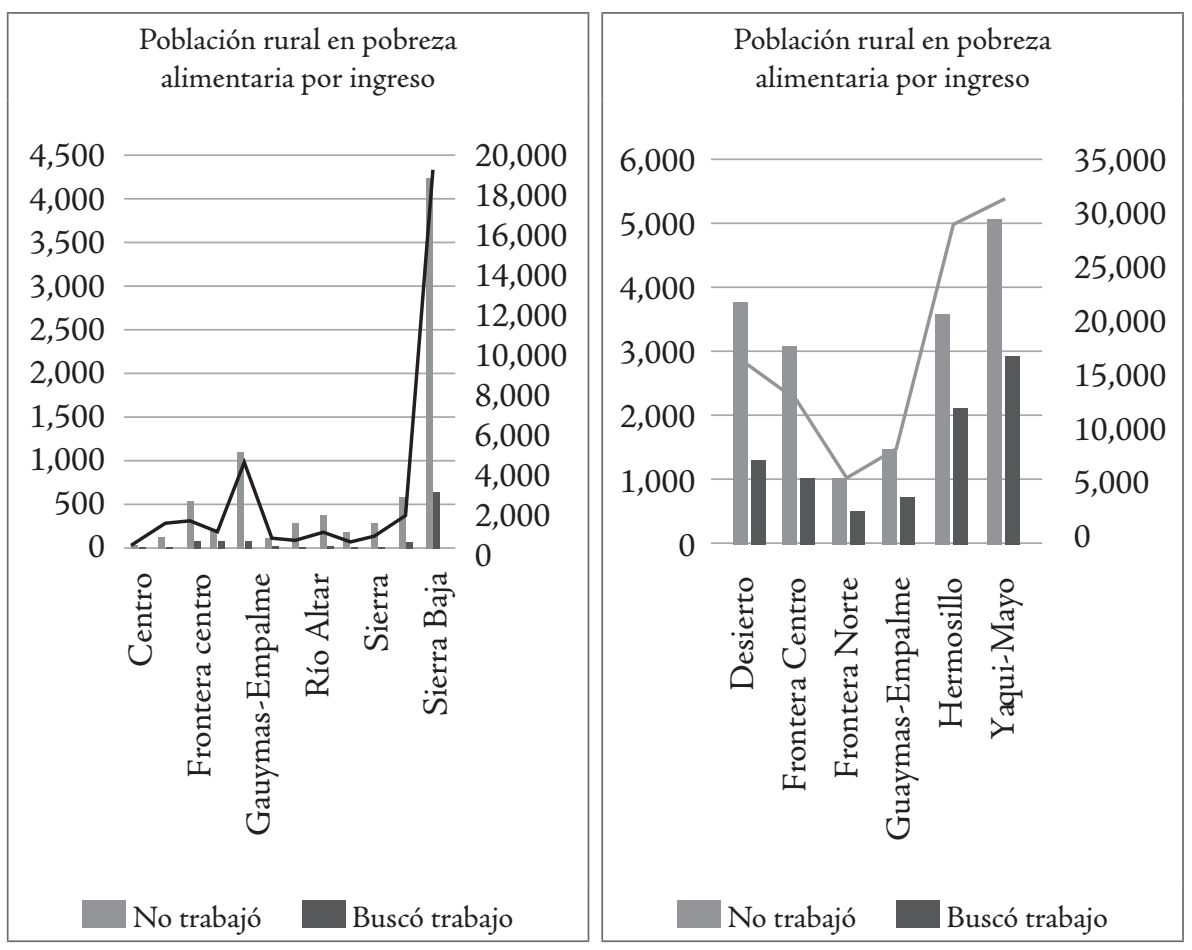

Fuente: Elaboración propia con base en la EIC-2015, INEGI.

La teoría del capital humano de Schultz (196I) y Becker (1962) señala que los conocimientos y las habilidades adquiridos con el estudio y la experiencia son factores esenciales del desarrollo humano y del crecimiento económico y explican en gran parte las diferencias en el ingreso entre las personas; sin embargo, el planteamiento de las capacidades de Sen (1996 y 200I) se relaciona más con el tema de la pobreza al señalar que: "Si una persona, mejorando su educación, su salud y otros aspectos, puede ser más productiva en la fabricación de bienes es lógico esperar que 
Figura 4. Nivel de educación primaria según zona y Región, POR CONDICIÓN DE POBREZA ALIMENTARIA, EN SONORA 2015.

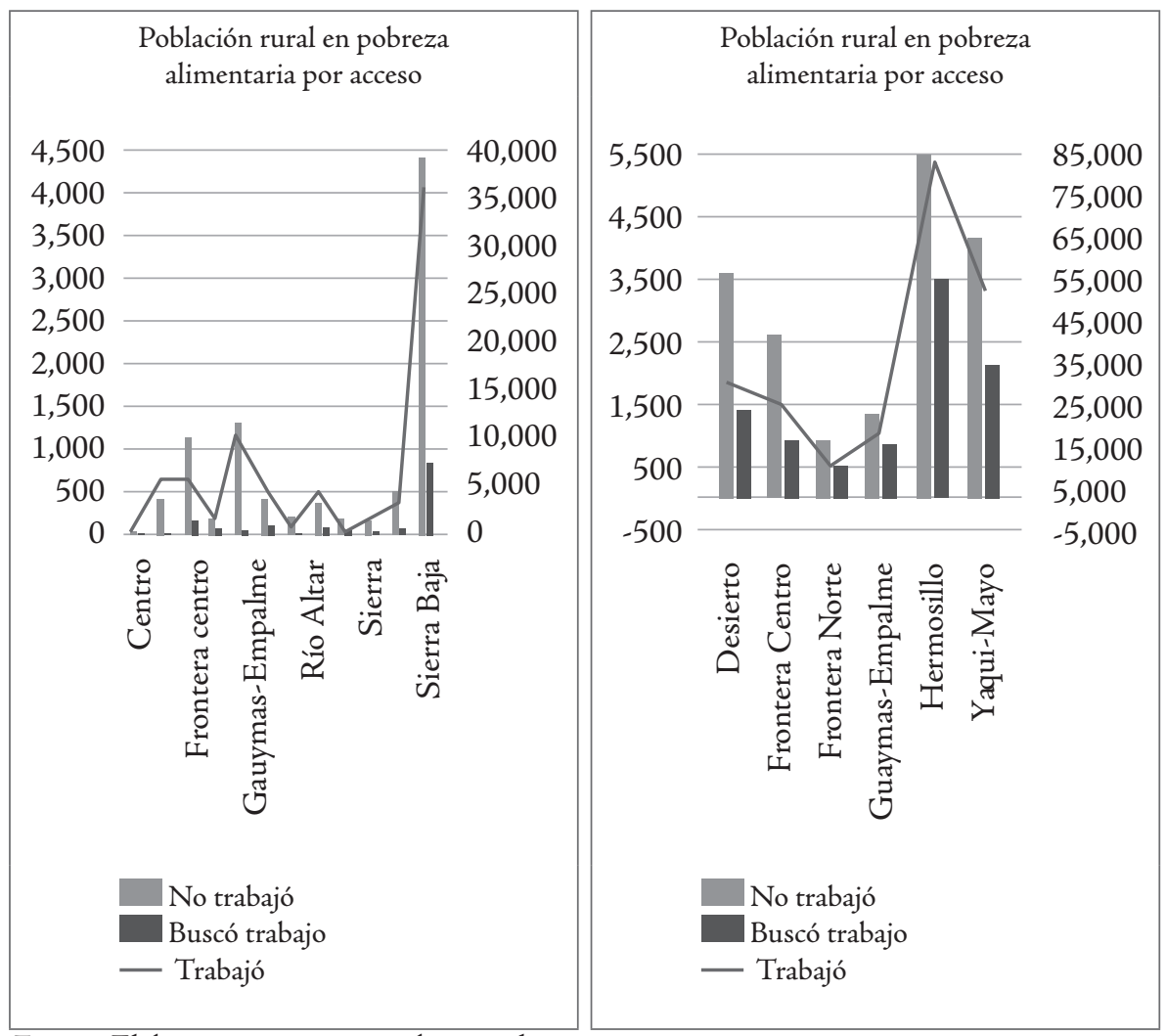

Fuente: Elaboración propia con base en la EIC-20I5, INEGI.

gracias a estos medios también tenga más posibilidades y libertad para desarrollar una mejor calidad de vida".

Con base en estos planteamientos se puede elaborar la hipótesis que expresa la relación inversa entre nivel educativo y los índices de pobreza alimentaria e inseguridad y el pertenecer a un grupo étnico; por el contrario, se espera una relación directa con la pertenencia a un sistema de salud y seguridad social, así como tener derecho a pensión, aspectos contemplados en esta investigación, que probaremos con los resultados para las distintas regiones que integran al estado de Sonora. 


\section{Vulnerabilidad por falta de escolaridad}

La educación se considera el principal elemento para la formación de capital humano en la medida en que determina la productividad y el bienestar de los individuos en una sociedad, por lo cual algunos estudios han concluido que los niveles de instrucción primaria y secundaria tienen un efecto significativo en el crecimiento de países en vías de desarrollo y la educación superior en las economías desarrolladas (Psacharopoulos y Woodhall, 1987).

El efecto de la educación en las regiones y zonas de Sonora la hemos separado en cuatro secciones: primaria, secundaria, preparatoria y licenciatura. La primera se muestra en la figura 4, que corresponde al nivel primaria y refleja un acumulado de $32.7 \%$ de la población en pobreza alimentaria por ingreso y $31.24 \%$ por condición de acceso, muy por encima de los porcentajes del promedio de la entidad sin considerar el grado educativo de $20.7 \%$ y $27.88 \%$, respectivamente, lo que significa que las personas con nivel de primaria están en peor situación que las de los grados educativos subsecuentes, como era de esperarse según la hipótesis.

Por zona y región, la parte izquierda de la primera sección de la gráfica 4 hace alusión a la zona rural, en la que se ve que la concentración de población en PZAXACC alcanza su punto más alto en la región Yaqui-Mayo, con 31540 habitantes, donde hay 23 921 personas con nivel de primaria y experimentan PZAXY. En dicha condición de pobreza encontramos las regiones de Guaymas-Empalme, Sierra Baja, Desierto y Frontera Centro, en orden descendente; de la misma forma se ubican Guaymas-Empalme, Desierto, Frontera Centro, mientras que Hermosillo alcanza la mayor cantidad de pobres por ingreso en la zona urbana y la Yaqui-Mayo en pobreza por inseguridad.

La siguiente sección se refiere a las personas en el nivel de secundaria (figura 5), en donde la población afectada por la inseguridad rebasa los 50000 habitantes (30.48\%); en tanto que la población en pobreza alimentaria por ingreso alcanzó $31.99 \%$, también por encima del promedio de la población sin considerar el grado educativo. Respecto del análisis por zona y regiones, se repite prácticamente el patrón distributivo de educación primaria para la población en pobreza alimentaria por acceso; en la zona rural, la región Yaqui-Mayo concentra la mayor cantidad de personas en pobreza alimentaria por acceso e ingreso ( 30378 y 22519 , respectivamente), seguida por Guaymas-Empalme con 7261 habitantes en pobreza alimentaria por acceso y 4629 por ingreso, y después las regiones Frontera Centro, Desierto y Hermosillo. 
Figura 5. Nivel de educación Secundaria Según zona y Región, por CONDICIÓN DE POBREZa Alimentaria, EN Sonora 2015.
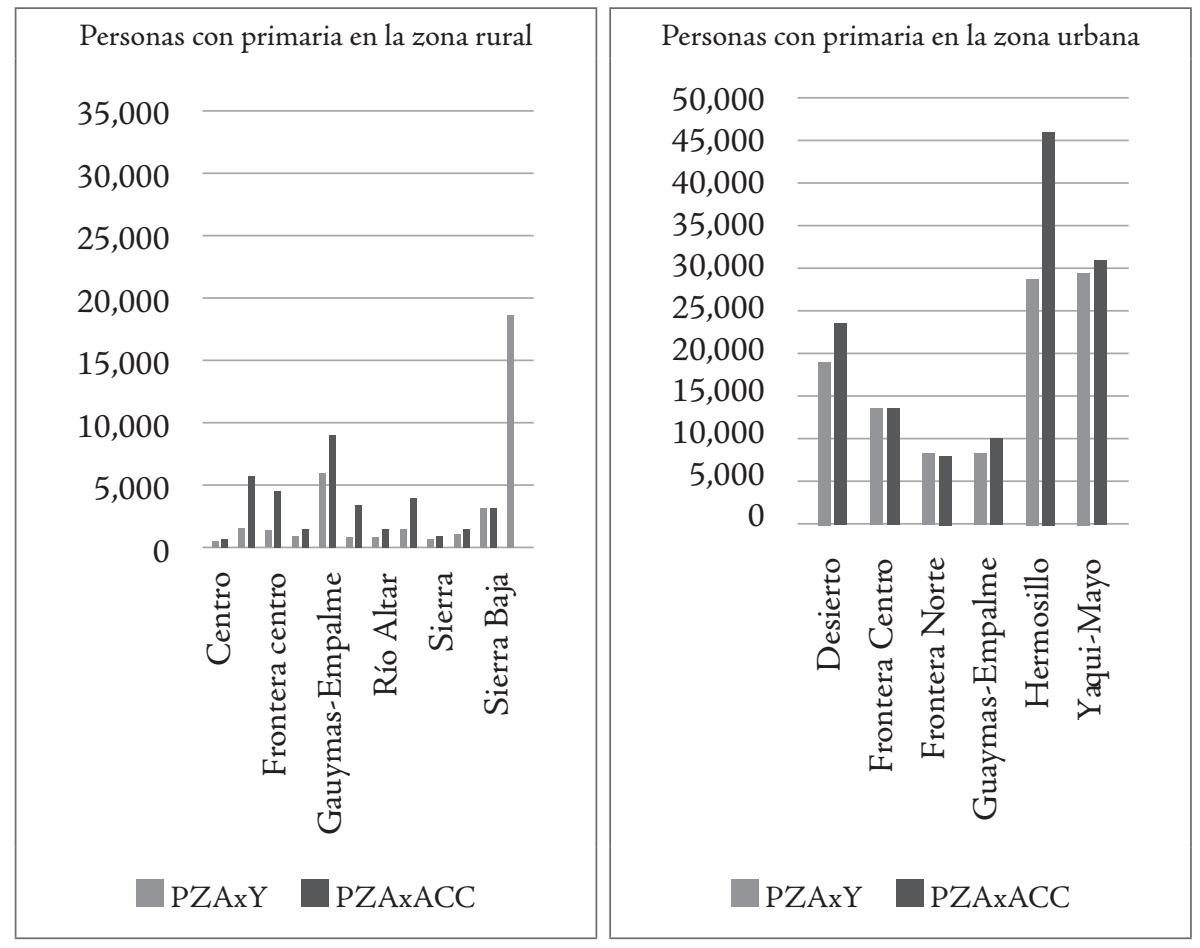

Fuente: Elaboración propia con base en la EIC-20I5, INEGI.

En lo que concierne a la zona urbana podemos observar seis regiones: Hermosillo (53212), Yaqui-Mayo, Desierto, Frontera Centro, Guaymas-Empalme y Frontera Norte, en orden descendente de acuerdo con la población en pobreza alimentaria por acceso; mientras que por ingreso se ubican de mayor a menor la región Yaqui-Mayo (33 019), Hermosillo, Desierto, Frontera Centro, Guaymas-Empalme y Frontera Norte.

Las personas con preparatoria inmersas en dicha problemática representan $15.52 \%$ por ingreso y $13.13 \%$ por acceso, y su distribución regional se repite como en las dos anteriores (figura 6); aunque referida a una población de menor magnitud en 
Figura 6. Nivel de educación preparatoria según zona y Región, por CONDICIÓN DE POBREZA ALIMENTARIA, EN SONORA 2015.

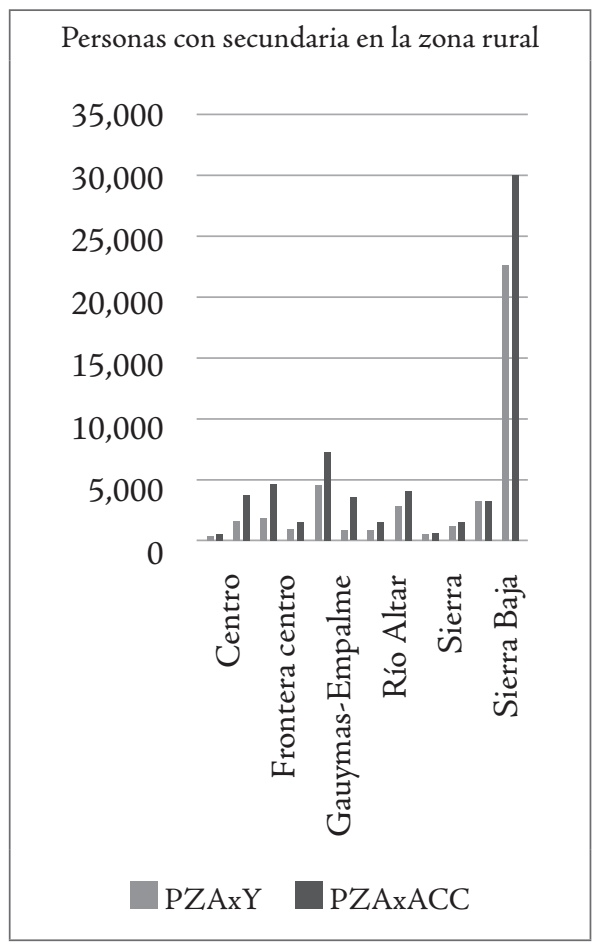

Personas con secundaria en la zona urbana
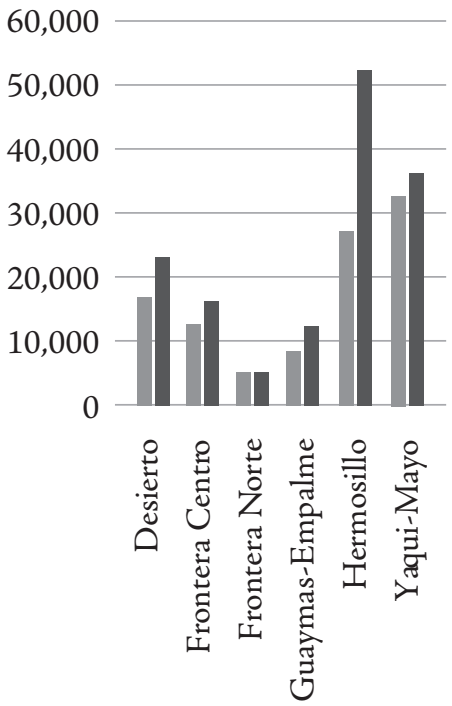

PZAxY

PZAxACC

Fuente: Elaboración propia con base en la EIC-20I5, INEGI.

la zona rural, la región Yaqui-Mayo destaca nuevamente (con I0 544 habitantes en PZAXACC y 8370 en PZAXY) en el primer lugar; también encontramos la de Sierra Baja como la de mayor participación en las regiones exclusivas del área rural.

En la página anterior (en la figura 6) se señalan las regiones urbanas. La de Hermosillo encabeza la lista con personas en pobreza alimentaria por acceso con preparatoria, seguida por las regiones del Yaqui-Mayo, Desierto, Frontera Centro, Guaymas-Empalme y Frontera Norte; mientras que las personas en pobreza alimentaria por ingreso la lidera la región Yaqui-Mayo, seguida por Hermosillo, Desierto, Frontera Centro, Frontera Norte y Guaymas-Empalme, respetando el 
Figura 7. Nivel de educación de licenciatura según zona y Región, POR CONDICIÓN DE POBREZA ALIMENTARIA, EN SONORA 2015.

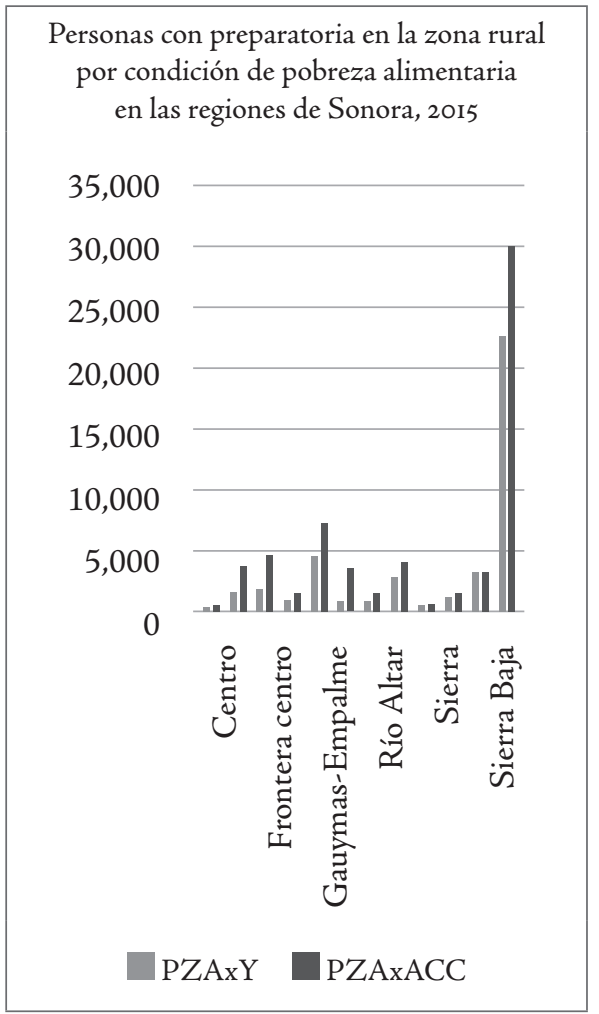

Personas con preparatoria en la zona urbana por condición de pobreza alimentaria en las regiones de Sonora, 2015

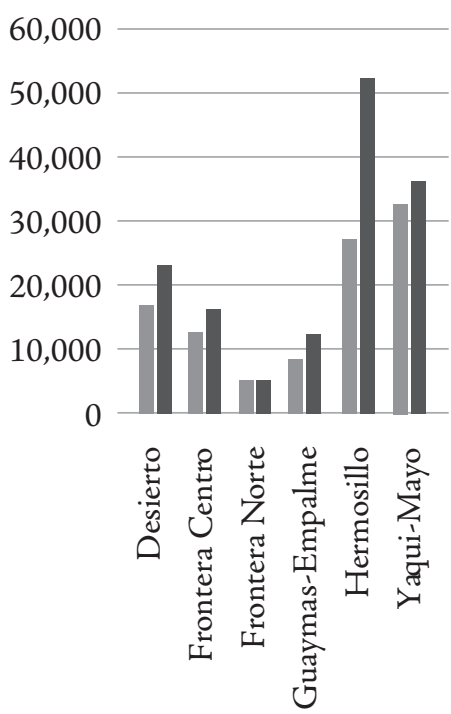

PZAxY $\square$ PZAxACC

Fuente: Elaboración propia con base en la EIC-2015, INEGI.

orden acostumbrado de mayor a menor. Se pone énfasis en que la región YaquiMayo de manera conjunta (urbana y rural) contiene poco más de 29000 personas en PZAXACC y alrededor de 24000 en PZAXY.

Las regiones dentro de la zona urbana expresadas en la parte derecha muestra a la de Hermosillo en el primerísimo lugar en ambas dimensiones de pobreza alimentaria (por acceso e ingreso), seguida por Yaqui-Mayo, Desierto, Frontera Centro, Guaymas-Empalme y Frontera Norte. 
Finalmente, la regionalización de la pobreza alimentaria en Sonora por nivel educativo nos muestra la región Yaqui-Mayo como la más afectada en los niveles de primaria, secundaria y preparatoria en conjunto (zona urbana y rural), mientras que en el de licenciatura (figura 7), lo es solo por ingreso, seguida por la región Hermosillo, siendo esta ultima la que registra el mayor número de personas en pobreza alimentaria por acceso con nivel de licenciatura.

De acuerdo con la literatura se podría interpretar que la mayor concentración porcentual de la población en pobreza alimentaria por ingreso en los niveles educativos de primaria $(32.70 \%$ ) y secundaria $(30.48 \%)$, y el menor porcentaje de pobres en el nivel medio superior (12.52\%) y superior $(4.64 \%$ ) corresponden al proceso de desarrollo económico de un país tercermundista. Estos resultados implican la relación que a menor nivel educativo el rendimiento del capital humano es bajo, de ahí los menores ingresos, lo que probaría la hipótesis del capital humano de Schultz (1960) cuya consecuencia para nuestra investigación, en línea también con la teoría de las capacidades básicas de Sen (1996), es que una política pública que pretenda ser exitosa en combatir la pobreza debe promover el aumento en el nivel educativo y mejorar su calidad, como condición necesaria para acceder a mejores empleos, aumentar los ingresos y salir de la pobreza.

\section{Vulnerabilidad por carencia de seguridad social}

Ya vimos cómo el nivel educativo se torna hasta cierto grado favorable para salir de la trampa de la pobreza alimentaria. Sin embargo, no lo es todo y tampoco suficiente. A continuación analizaremos lo vulnerable de la población ocupada, que además de ser pobre es vulnerable por carecer de seguridad social y corre el riesgo de empobrecerse más con las recesiones y crisis. La figura 8 indica por sí sola que existe un mayor número de población ocupada sin prestaciones sociales en la zona urbana, donde resalta la región Hermosillo en primer lugar tanto con personas en pobreza alimentaria por acceso como por ingreso, seguida de Yaqui-Mayo, luego Desierto, después Frontera Centro, posteriormente Guaymas-Empalme y finalmente Frontera Norte; ellas captan $36.63 \%$ y $30.86 \%$ del total de la población ocupada en pobreza alimentaria por ingreso y acceso. Al considerar la zona rural se vislumbra un cambio jerárquico donde la región Hermosillo cede el primer lugar a la Yaqui-Mayo, manteniéndose en tercera posición Desierto, seguida por Guaymas-Empalme, después Frontera Centro y luego Frontera Norte, dentro de la dimensión de pobreza alimentaria por ingreso; en lo referente al acceso se mantiene en el mismo orden 
Figura 8. Población ocupada sin prestaciones sociales por zona, SEgún CONDICIÓN DE POBREZa ALIMENTARIA Y REgIón EN SONORA, 2015.
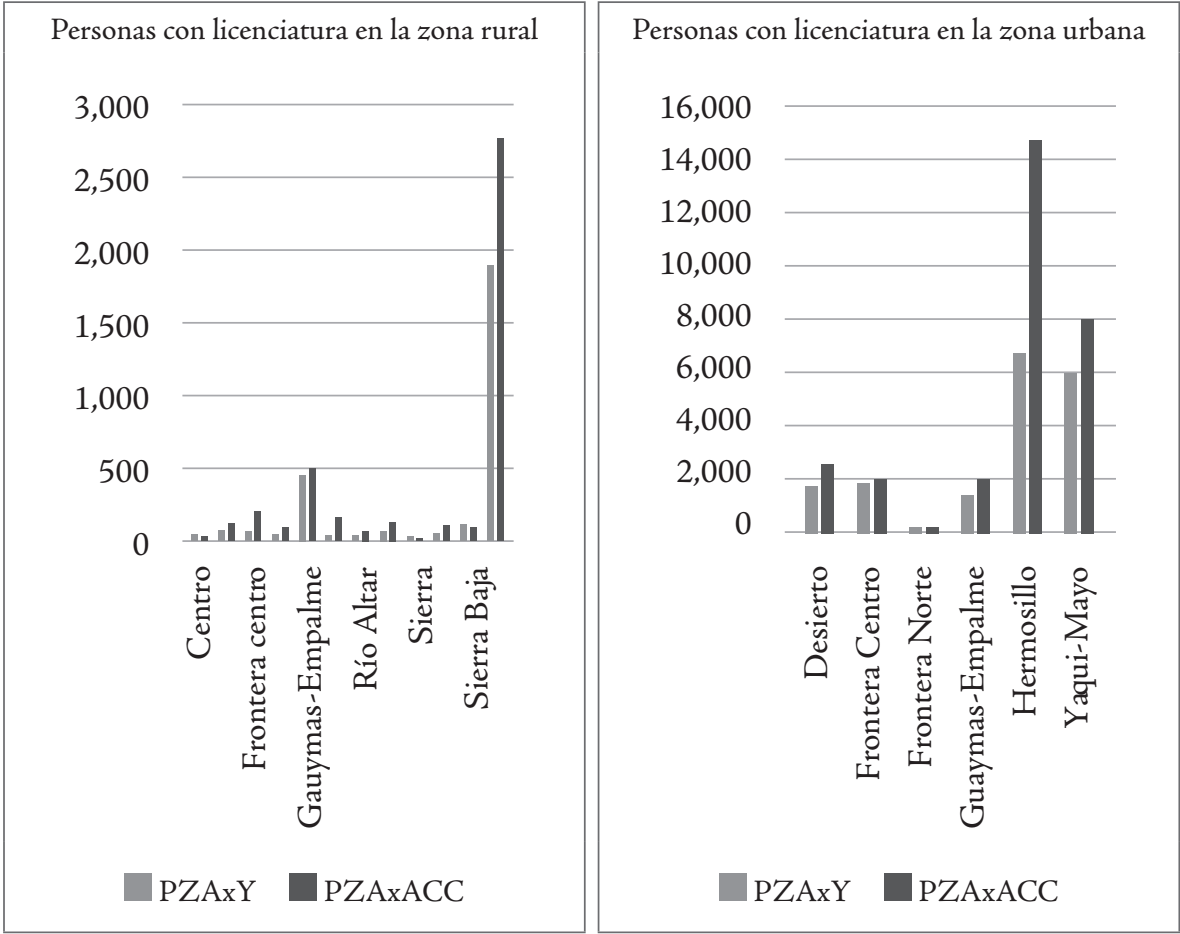

Fuente: Elaboración propia con base en la EIC-20I5, INEGI.

anterior: Yaqui-Mayo, Hermosillo, Desierto, Guaymas-Empalme, Frontera Centro y Frontera Norte. En su carácter conjunto dichas regiones representan $93.77 \%$ y 93.91\%, respectivamente, de la población ocupada sin prestaciones sociales en condición de pobreza alimentaria por ingreso y acceso.

Es importante mencionar que la población ocupada sin prestaciones sociales por región registrada en esta figura representa $54.87 \%$ y $47.78 \%$ del total de la población ocupada en condiciones de pobreza alimentaria por ingreso y acceso, proporcionalmente; por lo tanto, se habla de $50 \%$ de vulnerabilidad por prestaciones sociales. 
No debemos soslayar la zona rural, ya que representa $33.25 \%$ y $35.41 \%$ de la población ocupada sin prestaciones sociales en la dimensión de pobreza alimentaria por ingreso y acceso. Al considerar estrictamente las regiones rurales sin participación urbana estas significan $18.75 \%$ y $17.20 \%$, en el siguiente orden, de mayor a menor: Sierra Baja, Río San Miguel, Sierra Alta, Río Altar, Sierra y Centro en condición de pobreza por ingreso; mientras que por acceso se ubica en primera posición Río San Miguel, seguida por Sierra Baja, Sierra Alta, Río Altar, Sierra y Centro.

Definitivamente, las 78312 personas ocupadas sin prestaciones sociales en condición de pobreza alimentaria por ingreso y las 136316 en condición de pobreza alimentaria por acceso en su carácter conjunto agravarían más su problemática si por cuestiones de salud, accidente, incapacidad o cualquier imprevisto se vieran impedidas de continuar trabajando. Es así que no debemos perder de vista las regiones Yaqui-Mayo, Hermosillo, Desierto, Guaymas-Empalme, Frontera Centro y Frontera Norte que por orden jerárquico y acumulado de su zona rural son las que más población vulnerable registran.

\section{Vulnerabilidad por pensión insuficiente}

Un hecho que afecta el desarrollo de las regiones es el envejecimiento de su población y si agregamos a aquellas personas que aun con la pensión y/o jubilación bien ganada por su tiempo laborado no pueden escapar de la pobreza alimentaria, entonces esto se torna más delicado. La figura 9 alude a la distribución regional de la población jubilada o pensionada por zona rural (a la izquierda del gráfico) urbana (a la derecha). Cabe aclarar que solo se ha graficado a la población que recibe alguna pensión y/o jubilación (no la totalidad de la población que debería recibirla por su edad) y que se encuentra en pobreza alimentaria por ingreso o acceso. De un total de 45208 personas que reciben esta prestación, $28 \%$ se encuentran en pobreza alimentaria por ingreso y $20.21 \%$ de ellos registran pobreza alimentaria por acceso.

En la zona urbana, la región Yaqui-Mayo figura en primera posición, seguida por Hermosillo, Guaymas-Empalme, Desierto, posteriormente Frontera Centro y en último lugar la región Frontera Norte; este orden se mantiene tanto para la PZAXY como para la PZAXACC. Ya poseemos el conocimiento a priori de que en estas regiones coexiste población en la zona rural, la cual en su conjunto representa $98.52 \%$ y $98.16 \%$, respectivamente, para cada dimensión de pobreza alimentaria, manteniendo el mismo orden jerárquico regional. 
Figura 9. Población jubilada o Pensionada por zona, Según Condición De pobreza alimentaria y región en Sonora, 2015.

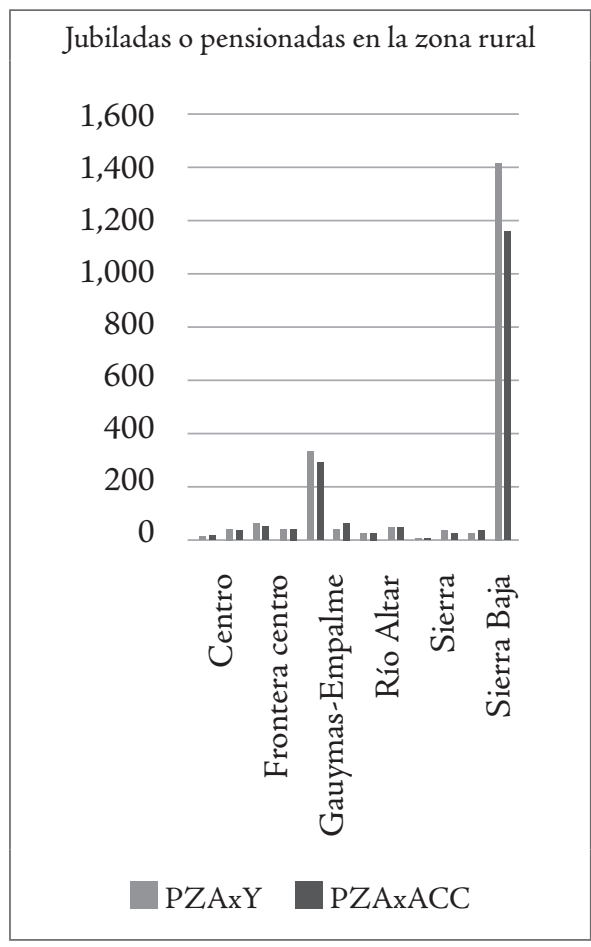

Jubiladas o pensionadas en la zona urbana

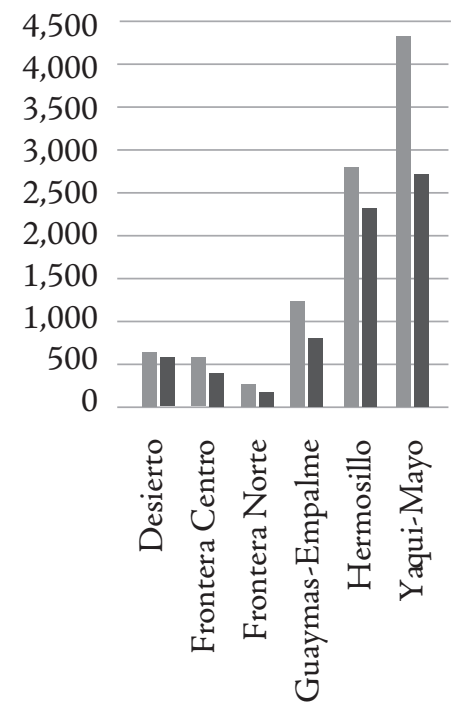

PZAxY $\square$ PZAxACC

Fuente: Elaboración propia con base en la EIC-20I5, INEGI.

Al comparar la zona rural al interior de sus regiones descubrimos que su totalidad representa $17.32 \%$ y $20.10 \%$ en pobreza alimentaria por ingreso y acceso, proporcional al total de personas que reciben pensiones o jubilaciones, mientras que las regiones exclusivamente rurales como Río San Miguel, Sierra Alta, Río Altar, Sierra Baja, Sierra y Centro representan $8.23 \%$ y $9.15 \%$ de las personas que reciben este apoyo y se encuentran en pobreza alimentaria por ingreso y acceso mutuamente; por cierto, la región Sierra Baja es la única que manifiesta un mayor número de personas en la dimensión de acceso que por ingreso. 
Figura io. Población indígena Por zona, SEgún CONDición de POBREZA alimentaria en las regiones de Sonora, 2015.
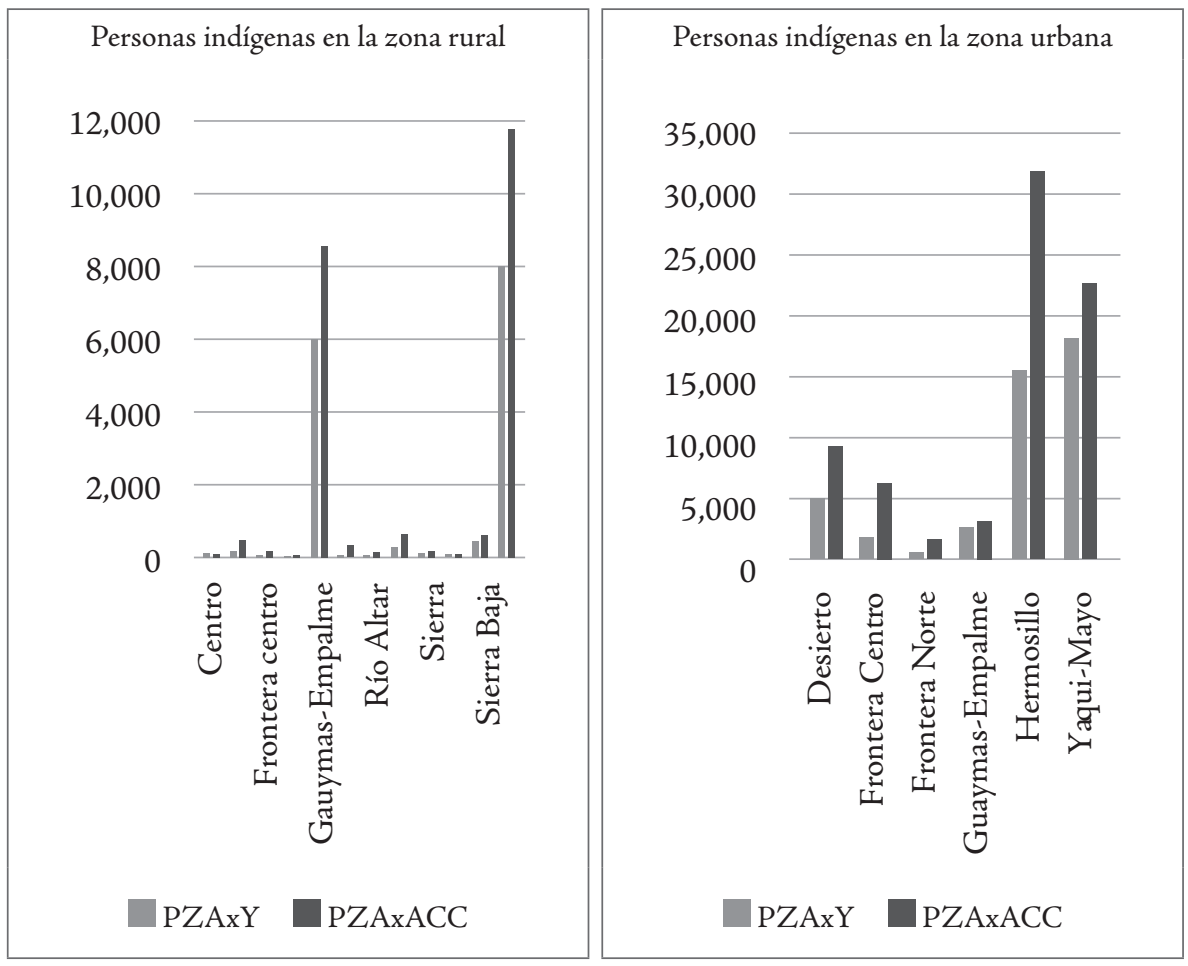

Fuente: Elaboración propia con base en la EIC-20I5, INEGI.

Vulnerabilidad por rasgos fenotípicos o pertenencia a grupo indígena

Otro aspecto relevante es el de la cultura indígena, debido a que existe cierto grado de discriminación hacia dicho estrato de población. Según McConell et al., (2007), "existe discriminación económica cuando individuos pertenecientes a grupos minoritarios reciben un trato diferenciado e inferior con respecto a los pertenecientes a grupos mayoritarios", esto reviste singular importancia; por lo tanto, se hace necesario disponer de información relacionada con su distribución en las distintas regiones del Estado. 
La figura Io integra este estrato poblacional por zona, región y dimensión de pobreza alimentaria. Esto deriva en 55,168 personas que se consideran indígenas dentro de nuestro universo de estudio, el $35.97 \%$ de ellos se encuentran en pobreza alimentaria por ingresos, mientras que el $56.95 \%$ lo está por acceso.

No es de sorprender que en la figura anterior se registre una mayor escala poblacional en la zona rural (a la izquierda) que la urbana (de la derecha); porque las regiones Yaqui-Mayo y Guaymas-Empalme, con una gran zona rural, son el asiento del mayor grupo indígena: la tribu yaqui, seguida por los mayos. Estas dos regiones comprenden $90.85 \%$ y $86.51 \%$ de personas en pobreza alimentaria por ingreso y acceso, respectivamente, mientras que las regiones meramente rurales (Centro, Río Altar, Río San Miguel, Sierra, Sierra Baja y Sierra Alta) representan 7 \% y 8.2 \% de la población en pobreza alimentaria por ingreso y acceso.

Incluso las regiones Centro y Sierra Baja apenas si registran $3 \%$ y $5 \%$ de personas indígenas en pobreza alimentaria e inseguridad. Los seris, con su asentamiento en la región rural de Hermosillo, viven la gran mayoría en pobreza, si bien es un grupo mucho menor al de los asentados en el sur de Sonora arriba mencionados, e incluso al de la Frontera con asentamiento de los pápagos y pimas.

Para finalizar, es importante decir que esta situación de los indígenas de Sonora confirma la hipótesis referida a la vulnerabilidad de los grupos indígenas respecto de quienes no lo son, lo cual los predispone a vivir mayormente en pobreza.

\section{Conclusiones y recomendaciones}

Al establecer la relación entre pobreza y alimentación se llegó al concepto de pobreza alimentaria, que ha servido para evaluar, a la vez, los logros en el combate a la pobreza, así como los referidos a la inseguridad alimentaria. Es en ese marco que este trabajo centra sus objetivos en conocer y medir la pobreza alimentaria acudiendo a los dos criterios antes señalados con respecto al ingreso y al acceso o inseguridad alimentaria, así como mostrar y exponer la vulnerabilidad en que se encuentra la población en pobreza alimentaria de Sonora y sus i2 regiones.

Los resultados de las estimaciones muestran que $20.70 \%$ de la población en pobreza alimentaria por ingresos resulta inferior a $27.88 \%$ que representa la población en pobreza alimentaria por acceso en Sonora, dando respuesta a la primera interrogante.

Era de esperarse que a mayor concentración de población por región, esta reflejara el mismo peso relativo de la población en pobreza alimentaria por ingreso; 
sin embargo, la región Yaqui-Mayo adquiere el primer lugar con 36.41 \%, muy por arriba de la región Hermosillo, que concentra a $20.95 \%$ de sus habitantes en esta condición, cuando es la que reúne 32.26 \% de la población total en el Estado; no obstante, las regiones Desierto, Frontera Centro, Guaymas-Empalme y Frontera Norte conservan esa representatividad y ese orden jerárquico poblacional, al igual que las de Centro, Sierra, Río Altar, Sierra Alta, Río San Miguel y Sierra Baja con menor representatividad y se enlistan de menor a mayor, con respecto a la población en pobreza e inseguridad alimentaria.

También encontramos que la vulnerabilidad de la población en pobreza alimentaria no se restringe a aquellos con menores niveles de educación y ausencia de seguridad social, sino que afecta a los pensionados y jubilados que alcanzan alrededor de $20 \%$ en condición de PZAXY y PZAXACC; además, la concentración porcentual de la población en pobreza alimentaria en los niveles educativos de primaria (32.70\%) y secundaria $(30.48 \%)$ corresponden al proceso de desarrollo económico de un país tercermundista; sin embargo, la interrelación existente del nivel medio superior ( $12.52 \%)$ y superior $(4.64 \%)$ con la pobreza alimentaria presente por regiones dista mucho de explicar el diferencial de lo que es a lo que debería ser.

Pudimos probar que los mayores índices de pobreza alimentaria e inseguridad se corresponden con los grupos de menor nivel educativo en los que el rendimiento del capital humano es bajo, y los ingresos son menores, en consonancia con la hipótesis del capital humano de Schultz (1960), cuya consecuencia para nuestra investigación, en línea también con la teoría de las capacidades básicas de Sen (1996), es que una política pública que pretenda ser exitosa en combatir la pobreza debe promover el aumento en el nivel educativo y mejorar su calidad, como condición necesaria para acceder a mejores empleos, aumentar los ingresos y salir de la pobreza.

Es importante señalar la situación que mantienen los jubilados, pensionados e indígenas de Sonora, con quienes se confirma la vulnerabilidad de los grupos minoritarios respecto de quienes no lo son, y que los predispone a vivir mayormente en pobreza.

Es por ello que recomendamos redoblar los esfuerzos de inversión directa para disminuir el flagelo de la pobreza alimentaria en la zona rural de Sonora y trabajar directamente en las regiones Yaqui-Mayo, Guaymas-Empalme y Sierra Baja, por observar altos índices de pobreza alimentaria e inseguridad y las elevadas concentraciones de la población. En segundo término se halla la necesidad de atender a los pobres de las regiones urbanas de Hermosillo y las regiones fronterizas.

En atención a esta recomendación y como segunda opción deberá apoyarse en los resultados que arrojaron el resto de variables utilizadas para captar la vulnera- 
bilidad de las regiones en este trabajo (la educación, la condición de actividad, la cultura, el género, la incapacidad física, entre otras) en las que se encontró una alta vulnerabilidad prácticamente en las mismas regiones arriba señaladas.

Por último, y como segunda etapa para erradicar la pobreza alimentaria en Sonora, se debe procurar el incremento en el ingreso salarial, especialmente proteger el valor real del salario mínimo regional; elevar el gasto social y la cobertura del sistema de pensiones en el ámbito urbano y rural; establecer las transferencias monetarias suficientes y directas para su erradicación; fortalecer la dimensión productiva de la zona rural, especialmente la agricultura de subsistencia o agricultura familiar; incrementar la creación de empleos formales que garanticen y permitan a las familias romper con la trampa de la pobreza.

\section{Bibliografía}

Alkire, Sabina y James Foster (20II), "Counting and multidimensional poverty measurement", Journal of Public Economics 95 (7-8), agosto, pp. 476-487, en $<$ http://dx.doi.org/I0.I0I6/j.jpubeco.2010.11.006 >.

Becker, G. (1962), "Investment in Human Capital: a Theoretical Analysis", The Journal of Political Economy, io, pp. 9-49.

Camberos, Mario (2000), "La seguridad alimentaria de México en el año 2030", Ciencia Ergo Sum, 7(I), marzo, disponible en <http://www.redalyc.org/ articulo.oa?id $=10401706>$.

Camberos, Mario; Huesca, Luis y Bracamontes, Joaquín (2013), "La pobreza en la frontera Arizona-Sonora: medición, causas y soluciones", en G. Córdova B., J. Dutram H., Blanca E. Lara E. y J. G. Rodriguez (coords.), Desarrollo bumano y transfronterizo, México: Colegio de Sonora/Colegio de la Frontera Norte/Universidad de Sonora/Universidad Estatal de Sonora, pp. 25-43.

Camberos, Mario; Gutiérrez, Luis y Huesca, Luis (2012),"Un criterio de ordenamiento para la atención de la pobreza y el rezago social en Sonora", en V. Félix y Gustavo y Gilberto Aboites M., Dimensiones socioeconómicas de la pobreza en México, México: Plaza y Valdés, pp. 289-320.

Camberos, Mario; Bracamontes, Joaquín y Gutiérrez, Luis (2012), "Aplicación de una metodología para medir la pobreza, estimar sus costos y elaborar criterios de asignación de recursos para combatirla”, en V. Félix y Gus- 
tavo y Gilberto Aboites M., Dimensiones socioeconómicas de la pobreza en México, México: Plaza y Valdés, pp., 253-287.

Camberos, Mario; Genesta, María Antonieta y Huesca, Luis (1996),"Pobreza regional en Sonora: tendencias recientes, recursos necesarios para combatirla y perspectivas para el año 200o", Investigación Económica, LVI(215), enero/ marzo, México: Universidad Nacional Autónoma de México,, pp. 163-199.

Camberos, Mario; Genesta, María Antonieta y Huesca, Luis (1994), "La pobreza en Sonora: los límites a la modernización”, Estudios Sociales, v(9), enero-junio, pp. 167-197.

Castillo Fernández, Dídimo y Jorge Arzate Salgado (2013), "Crisis económica, pobreza y política social en México", Aportes, Revista de la Facultad de Economía, xviri(47), enero-abril.

Castro Robles, Abel Ernesto (2016), El trazo de la pobreza e inseguridad alimentaria: un ejercicio de referenciación bidimensional en Sonora, 2015, tesis de maestría inédita, Centro de Investigación en Alimentación y Desarrollo (CIAD).

Comité Científico de la Escala Latinoamericana de Seguridad Alimentaria (2012), Escala Latinoamericana de Seguridad Alimentaria (eLCSA): Manual de uso y aplicaciones, Santiago de Chile: Organización de las Naciones Unidas para la Agricultura y la Alimentación.

Consejo Nacional de Evaluación de la Política de Desarrollo Social (coneval) (2010), La pobreza por ingresos en México, México: Coneval.

Food and Agriculture Organization (FAO) (2002), La reducción de la pobreza y el hambre: la función fundamental de la financiación de la alimentación, la agricultura el desarrollo rural. Roma, Febrero de 2002; Documento preparado para la Conferencia Internacional sobre la Financiación para el Desarrollo Monterrey, México: 18-22 de Marzo de 2002.

Food and Agriculture Organization (FAO) (2005), Departamento de estadísticas de la FAo (FAOSTAT, por su sigla en inglés).

Food and Agriculture Organization (FAO) (20IO), Departamento de estadísticas de la FAo (FAOSTAT, por su sigla en inglés).

Food and Agriculture Organization (FAO) (20II), Seguridad Alimentaria: Nutrición para la salud y el desarrollo.

Food and Agriculture Organization (FAO) (2015), Cumplimiento de los objetivos internacionales para 2015 en relación con el hambre: balance de los desiguales progresos. 
Foster, James, Joel Greer y Erik Thorbecke (1984), "Notes and comments: a class of decomposable poverty measures", Econométrica, 52(3), mayo, pp. 76I-766.

Gobierno de la República Mexicana, Programa Nacional México sin Hambre (2014), Plan Nacional de Desarrollo, 2013-2018", Logros 2014, Especial.

Gómez Bruera, Hernán F. (2013), Desafíos para garantizar el derecho a la alimentación: las experiencias contrastantes de México y Brasil. México: CEPAL. Instituto Nacional de Ciencias Médicas y Nutrición Salvador Zubirán, Sociedad Latinoamericana de Nutrición (2003), México.

Instituto Nacional de Estadística y Geografía (INEGI). Encuesta Intercensal (2015), Síntesis metodológica y conceptual. Aguascalientes, México: INEGI, disponible en <encuestas.inegi.org $>$.

McConell, Campbell R., Stanley L.Brue y David A. Macpeherson (2007), Economía Laboral, [7a. ed.], México: McGraw-Hill.

Minor Campa, Enrique E. y Rodrigo Balcázar Aranda (2014), "Elaboración de canastas alimentarias regionales para la medición de la pobreza en México", en Mercado de trabajo, desarrollo regional y políticas públicas, México: Universidad Autónoma de Baja California Sur.

Psacharopoulos, George y Mauren Wooddhall (1987), Educación para el desarrollo: un análisis de opciones de inversión, Madrid: Tecnos, Publicación del Banco Mundial, p. 42

Schultz, T. W. (1960), "Investment in Human Beings", suplemento del Jourrnal of Political Economy 70, octubre, pp. 50-79.

Sen, Amartya (1976), "Poverty: An Ordinal Approach to Measurement", Econometrica, 44(2), marzo, pp. 219-23I, en <http://www.jstor.org/stable/1912718> [consulta: 28/12/2015].

Sen, Amartya (1996), “Teoría de las capacidades”, en Nusbaum M. y A. K. Sen, Capacidad y Bienestar, México, Fondo de Cultura Económica.

Sen, Amartya (200I), Desarrollo y libertad, Bogotá: Planeta, p. 125.

\section{Consultas en internet}

$<$ http://www.ucm.es/info/solidarios/malnutricion_desnutricion.doc $>$ [consulta: 14/II/2014].

Informe de evaluación de la politica de desarrollo social en México, 2014, en <https:// issuu.com/politicaspublicas/docs/coneval_iepds_2014>. 
Resúmenes CURRICUlares

Abel Ernesto Castro Robles es licenciado en Economía por la Universidad de Sonora y Maestro en Desarrollo Regional CiAd. A.C. Su línea de investigación se define como Bienestar y Mercados Laborales. Entre sus publicaciones recientes se encuentran: Interacción ocupacional y Profesional en la Frontera Norte de México y Sonora Ante el Cambio Tecnológico (1998-2010), en "Mercado Laboral y Cambio Tecnológico en México: Tendencias, Sector y Regiones" y; La Sensibilidad del Mercado de Trabajo Regional de Sonora ante Cambios en el Crecimiento Económico 2000-2010, en "Mercado de trabajo, desarrollo regional y políticas públicas"

Mario Camberos Castro es licenciado en Economía por la Facultad de Economía de la Universidad de Guadalajara. Maestro en Ciencias Económicas, Facultad de Economía, Universidad Nacional Autónoma de México. Doctor en Ciencias Económicas, Universidad Autónoma de Baja California-Tijuana. También es candidato a doctor por la Universidad Autónoma Metropolitana-Iztapalapa. Su línea de investigación se centra en Política Económica, Bienestar, Mercados Laborales y evaluación de Políticas Públicas. Entre sus publicaciones recientes: "Is the Economic Convergence of Mexican States Possible?" Review on Global Economics, Life Science Global. E-ISSN: 1929-7092-16, pp. 48-58; "Riesgo de pobreza en las regiones mexicanas: Una estimación con un método que considera las deudas de los hogares." Revista Politicas Sociales Sectoriales. UANL, ISSN: 2395-8456, Agosto 20I5-Julio 2016/Año.2, No. 2. pp. 836-857.

Citar como: Castro Robles, Abel E. y Camberos Castro, Mario (2017), "Pobreza alimentaria: inseguridad y vulnerabilidad en las regiones de Sonora en 2015", Iztapalapa. Revista de Ciencias Sociales y Humanidades, núm. 83, año 38, julio-diciembre de 2017, ISSN: 2007-9176; pp. 43-73. Disponible en $<$ http://revistaiztapalapa.izt.uam.mx/index.php/izt/issue/archive $>$. 